\section{P101 THE LONG-TERM EXPERIENCE OF COPD PATIENTS TAUGHT PLB: A MIXED METHODOLOGICAL STUDY}

doi:10.1136/thoraxjnl-2012-202678.384

'SE Roberts, ${ }^{2} F M$ Schreuder, ${ }^{2} \mathrm{~T}$ Watson, ${ }^{3} \mathrm{M}$ Stern. 'Dept Physiotherapy, Whittington Health, London, United Kingdom; ${ }^{2}$ School of Health \& Emergency Professions, University of Hertfordshire, Hatfield, United Kingdom; ${ }^{3}$ Dept. Respiratory Medicine, Whittington Health, London, United Kingdom

Background Pursed lips breathing (PLB) is a breathing technique advocated for the treatment of exertional dyspnoea in COPD. Published studies to date have only reported on immediate or shortterm effects

Aim To investigate the long-term use of PLB in people with COPD trained to use this technique for dyspnoea management.

Method A purposive sample of 13 patients taught PLB 6-24 months previously were studied using semi-structured telephone interviews and a focus group. Where possible the technique of those reporting current PLB use was also observed.

Results 11 participants took part in the telephone interviews, mean age 64.6 yrs (SD11.81), mean $\mathrm{FEV}_{1} 44.3 \%$ predicted (SD 0.19). $5 / 11$ participated in the focus group and $6 / 11$ were observed performing PLB. Nine reported on-going use of PLB with 8 reporting definite benefit. Four distinct themes emerged from the analysis of the data: use of PLB when short of breath due to physical activity $(8 / 9)$, increased confidence and reduced panic (4/9), use as an exercise (3/9), use at night (3/9). Observed technique showed ongoing ability for PLB to reduce respiratory rate and increase oxygen saturation. Those that had discontinued PLB had done so because it didn't help (2) and they had forgotten or were too busy to continue. No substantial adverse effects were reported.

Conclusion This study investigated, for the first time, the long-term use of PLB by patients with COPD. $62 \%$ of patients studied reported benefit from PLB up to 24 months after learning the technique. The role of PLB in increasing patients' confidence in their ability to manage breathlessness and, use at night, are also novel findings.

\section{P102 POST-HOSPITALISATION OUTPATIENT PULMONARY REHABILITATION: A TRANSLATIONAL GAP?}

doi:10.1136/thoraxjnl-2012-202678.385

${ }^{1} \mathrm{SE}$ Jones, ${ }^{2} \mathrm{SA}$ Green, ${ }^{3} \mathrm{AL}$ Clark, ${ }^{4} \mathrm{MJ}$ Dickson, ${ }^{4} \mathrm{~A}-\mathrm{M}$ Nolan, ${ }^{4} \mathrm{C}$ Moloney, ${ }^{1} \mathrm{SSC}$ Kon, ${ }^{3} \mathrm{~J}$ Godden, ${ }^{2} \mathrm{C}$ Howe, ${ }^{4} \mathrm{BM}$ Haselden, ${ }^{3} \mathrm{~S}$ Fleming, ${ }^{1} \mathrm{WD}-\mathrm{C}$ Man. ${ }^{1}$ Respiratory Biomedical Research Unit, Royal Brompton and Harefield NHS Foundation Trust, Harefield, Middlesex, United Kingdom; ' ${ }^{2}$ NIHR CLAHRC for Northwest London, London, United Kingdom; ${ }^{3}$ Royal Brompton and Harefield NHS Foundation Trust, Harefield, Middlesex, United Kingdom, ${ }^{4}$ The Hillingdon Hospital NHS Foundation Trust, Middlesex, United Kingdom

Background Recent trials and meta-analyses of early posthospitalisation pulmonary rehabilitation (PR) in COPD have demonstrated improvements in exercise capacity, health-related quality of life and a reduction in hospital readmissions (Man et al., 2004; Seymour et al. 2010; Puhan et al. 2011). However anecdotal observation and evidence from recent trials suggest poor uptake of outpatient PR. The aim of the study was to map patient journeys to identify gaps or deficiencies in the referral pathway.

Methods All 224 patients discharged from Hillingdon Hospital following an acute exacerbation of COPD between November 2011 and May 2012 were included in the analysis. Referrals for post-exacerbation PR from Hillingdon Hospital were monitored during the same time period. A collaborative of 18 stakeholders from seven organisations across primary, secondary and community care services was convened and performed local process mapping. Structured telephone interviews were held with a convenience sample of 36 COPD patients who declined post-hospitalisation PR.
Results Despite excellent compliance with a COPD discharge bundle (95\%), only 63 (28\%) of the 224 discharges from Hillingdon Hospital were referred to the local PR provider. All referrals were offered initial assessment for PR within 2 weeks of discharge but 18 failed to attend on at least 2 occasions. A further 9 patients failed to start PR despite attending initial assessment. In total, only $36(16 \%)$ patients out of all hospital discharges over a 6 -month period started outpatient PR. The main reasons for patients declining outpatient PR were accessibility issues (40\%), commitment to PR "too timeconsuming" (20\%) or "too unwell" (13\%).

Conclusion Despite a strong evidence base, there is poor uptake of post-hospitalisation early PR. The majority of missed opportunities occur at the initial referral stage, although there is a significant drop-out even in those referred. Ongoing experience based design work will explore staff and patient attitudes that may influence referral and uptake rates.

\section{P103 CARDIOPULMONARY EXERCISE TESTING IN COPD: CYCLE ERGOMETRY OR TREADMILL WHICH IS BETTER?}

doi:10.1136/thoraxjn--2012-202678.386

Mir Shad Ali, S Joshi, Mandeep Singh, AS Sandhya, D Talwar. Metro centre for Respiratory diseases, Noida, INDIA

Introduction/Objectives Traditionally Cycle Ergometry is used for CPET to assess functional exercise capacity in COPD. However, walking is closely related to daily functional needs of COPD patients. The aim of this study was to evaluate the efficacy and tolerance of Naughton's treadmill protocol over Cycle Ergometry in COPD patients during maximal CPET.

Result All patients were able to complete the treadmill protocol in CPET, while on the Cycle Ergometry test it was symptom limited. The mean age was $58.0+10.1$ years with mean FEV1\% of $56.11+$ $26.2 \%$. VE, HR, VE/VCO2, VE/VO2, VD/VT and PETCO2 at the end of the exercise during treadmill and Cycle Ergometry were not statistically different. Hence all variables e.g. VO2 peak, VO2\% predicted, duration of exercise and VO2 at LT were comparable. The VO2 Peak during treadmill was significantly higher during treadmill as compared to Cycle Ergometry $(1347.5+308 \mathrm{ml} / \mathrm{min}$ vs. $1089.9+277.9 \mathrm{ml} / \mathrm{min}$ respectively; $\mathrm{p}=0.013)$. The duration of exercise was also significantly more during treadmill as compared to cycle ergometer $(12.6+3.8 \mathrm{~min}$. vs. $8.3+3.12 \mathrm{~min}$. respectively $p=0.002 ; p=0.68)$ although there was no significant difference in VO2 at LT $(41.3+14.1 \%$ during treadmill vs. $33.4+10.6 \%$ during cycle ergometry; p-value- 0.087).

Conclusion Patients performing CPET on treadmill as compared to Cycle Ergometry showed increase exercise capacity. Hence in Indian COPD subjects treadmill CPET may be better for functional assessment.

\section{P104 RESPONSE OF THE COPD ASSESSMENT TEST (CAT) TO PULMONARY REHABILITATION IN NON-COPD PATIENTS}

doi:10.1136/thoraxjnl-2012-202678.387

'SSC Kon, ${ }^{2} \mathrm{AL}$ Clark, ${ }^{2} \mathrm{D}$ Dilaver, ${ }^{2} \mathrm{MM}$ Peasey, ${ }^{1} \mathrm{JL}$ Canavan, ${ }^{1} \mathrm{SE}$ Jones, ${ }^{2} \mathrm{MGS} \mathrm{Ng}$ 'MS Patel, 'Ml Polkey, 'WDC Man. 'Respiratory Biomedical Research Unit, Royal Brompton \& Harefield NHS Foundation Trust, Harefield, Middlesex, United Kingdom; ${ }^{2}$ Harefield Pulmonary Rehabilitation Team, Middlesex, United Kingdom

Background The COPD (chronic obstructive pulmonary disease) assessment test (CAT) is a recently introduced, simple to use health status instrument, which takes less time to complete than betterestablished health status instruments (Jones PW et al 2009, Ringbaek $T$ et al 2012). In COPD patients, the CAT improves with pulmonary rehabilitation (PR) and correlates with improvements in longer established health status instruments such as the Chronic Respiratory 